\title{
CPTAC Baseline Medical Forms Terminology
}

National Cancer Institute

\section{Source}

National Cancer Institute. CPTAC Baseline Medical Forms Terminology. NCI Thesaurus. Code C156953.

Terminology used in support of the data collection efforts of the Clinical Proteomic Tumor Analysis Consortium (CPTAC) with the focus on obtaining data for baseline medical forms. 